\title{
Restrictions in the design of gear wheel components and drives for micro technology
}

\author{
A. Albers, N. Burkardt, J. Marz
}

\begin{abstract}
According to enhanced interest for use of micro mechanical gear drives increased requirements occur for the transmittable power output with at same time proceeding miniaturization. To meet these future necessities research trends lead to micro technical parts from metal and ceramic materials. Using these materials the design of the parts has to meet restrictions of several disciplines, predominantly manufacturing and moulding technologies.
\end{abstract}

\section{1}

\section{Introduction}

Micro mechanical components and systems are more and more of central interest. Up to now it is common to use assured manufacturing technologies nearly limited to the application of polymer materials. A special group of Karlsruhe research institutes within the DFG funded collaborative research centre (Sonderforschungsbereich) 'Development, production and quality assurance of primary shaped micro components from metallic and ceramic materials' tries to increase the focus on a broader range of steps in the product development process with its central task of micro primary shaping of metallic and ceramic parts.

As far as the design is concerned there exists a lack of systematically collected and edited know-how acquired during the concept and draft development of micro mechanical parts and systems which could be generalized so it can be used as a template for future product developments.

\section{2}

\section{Design facing technological restrictions}

To learn something about where the momentary technological possibilities and limits are, a micro planetary gear drive has been chosen to represent and examine the existing know-how of development, production and quality

Received: 10 August 2001/Accepted: 24 September 2001

\author{
A. Albers, N. Burkardt, J. Marz ( $₫)$ \\ Institute of Machine Design and \\ Mechanical Engineering, \\ University of Karlsruhe, \\ Kaiserstrasse 12, D-76128 Karlsruhe \\ Germany
}

This paper was presented at the Fourth International Workshop on High Aspect Ratio Microstructrue Technology HARMST 2001 in June 2001. assurance. This classical mechanical system has reached its so far highest degree of miniaturization within a three stage version with parts produced by LIGA in a polymer material [7].

\section{1}

\section{Manufacturing technologies}

Since the parts will be primary shaped the form tools have to be cut of high strength steel to ensure a high tool life for economic middle and large series production. Besides the moulding technologies micro moulding and micro powder injection moulding the micro milling represents momentarily the predominant restrictive technology according to the feasible miniaturization.

\section{2}

\section{Restrictive criteria}

Looking at the technological data and parameters of subsequent product development processes demands and restrictions occur for the design of primary shaped micro parts.

\section{Micro milling}

For reproducible cutting results the smallest tool utilized is a micro end mill with a shank diameter of 100 microns.

This sets the basis for minimum structure measurements. Due to a direct relation the realizable cutting depth can only be between two and three times the diameter. Moreover, the resulting rounding at the part contours have to be taken in account for the design of micro technical parts.

\section{Micro powder injection moulding}

To produce highly chargeable micro technical parts, materials such as metal and ceramic can multiplicate the product lifetime and enable the use in higher load ranges at all. In the case of the micro planetary gear the increased momentum due to the transmission ratio causes high friction and Hertz' pressure on the paired gear flanks. Therefore, the material of the paired gear partners have to be both the same. In a first step $\mathrm{ZrO}_{2}$ is chosen since it has proved material properties withstanding high pressure stress.

Zirconium dioxide powder is processed by mixing it with a binder getting a viscose feedstock and this being injection moulded. After debinding the primary shaped part passes through a sinter treatment shrinking by about $22 \%$. This linear shrinkage in all dimensions has to be assigned in the design of the cavity. 


\section{3}

\section{Micro planetary gear}

This concrete and specific micro technical device helps to investigate the differences to the commonly used macroscopic product development methodology. Once established new or adapted methodological procedures, effects and principles have to be verified at further applications to finally come up with a generalized method tool box for micro technology. Implementing a first design draft standardized design criteria and formula of the macro gear design theory $[1,5,6]$ are taken in account and unless otherwise provided transferred to the micro scale. To what extent this is acceptable will future applications show. At the moment, it helps to simplify the layout and calculation of the gear drive as a lot of interconnected gear parameters have to be looked at simultaneously. This lack of standardisation and experience in development means another important restriction in the design of micro technical parts and systems.

\section{3}

\section{Finding the optimal gear layout}

Following the detected restrictions on the part of the form tool manufacturing and moulding technologies it is searched for a design strategy to build up a planetary gear drive as small as possible. As mentioned above, the end mill diameter causing a tip rounding of 50 microns in radius at the tip of the tooth is the predominant restriction. To demonstrate the intensity of this phenomenon an initial design draft was constituted once without tip rounding and afterwards with tip rounding. The comparable parameter is the transverse contact ratio $(\varepsilon)$. To guarantee an undisturbed contact of the paired working surfaces the ratio should be greater or at least equal 1 .

By a systematically investigation of the different gear parameters the optimal layout successively could be carried out.

\section{1}

\section{Initial design draft}

As flank shape the involute toothing will be used with its along others well-known advantages to keep up a constant ratio of transmitted momentum and revolution when opposed to alternating centre distances and to be adaptable to special demands.

The planetary gear is designed in the structural shape of an AAI gear:

A single stage consists of three planet wheels arranged with an offset angle of $120^{\circ}$ around the central sun wheel while the ring gear is fixed in the housing. Working as an reduction gear the sun is powered and the outlet is realized by the planet carrier.

The module selected equals the end mill shank diameter of 100 microns. The number of teeth was determined to 12 for the sun, 10 for the planet and -33 for the ring gear. As far as the sun and planet are concerned the gearing lies significantly below the permissible practical limit for the minimum number of teeth respectively to DIN 867 [1]. To avoid undercut a positive addendum modification became necessary for both the sun and the planet to sustain an

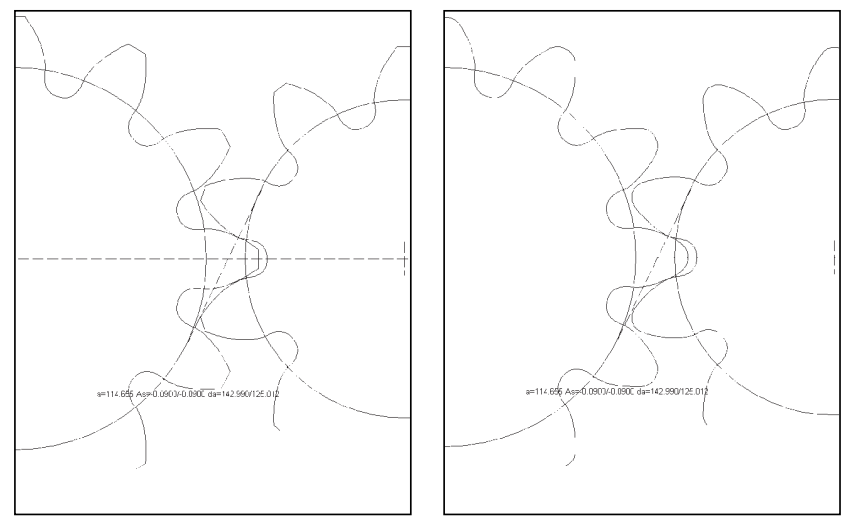

Fig. 1a, b. Focus on the contact area between sun and planet for the initial design draft. a Without tip rounding; $\mathbf{b}$ with tip rounding

appropriate tooth root strength while the demanded minimum transverse contact ratio is maintained.

Figure 1 shows a focus on the area of contact between the sun and the planet without and with a tip rounding caused by the end mill.

The version without the tip rounding matches a contact ratio of 1.2 , whereas the modified one falls dramatically below the minimum of 1 .

Following this knowledge the central design task expresses itself like this: find the smallest planetary gear by constituting around a 50 micron tip tooth rounding gear flanks with an appropriate length of working flank surface to ensure that minimum one tooth is in transverse contact all the time.

\section{2}

\section{Variation of gear pairs}

As the geometrical layout of a gear is determined by several parameters highly interconnected with each other it seems much too complicated to consider them all at once. To find an optimised planetary gear drive in the sense of highest miniaturization a systematically way of investigation has to been undergone.

Therefore, the system has to be reduced to a minimum amount of elements varying only a single parameter while preferably holding the rest fixed. After learning about the individual influence of each parameter on the subsystem the focus can be enlarged by the next more extensive subsystem using former tendencies to identify the startingpoint to optimise the higher level system. This step by step proceeding leads to the final optimised product.

The methodology behind this is that of a 'systematic variation of existing parameters' appendant to the group of systematic methods.

As a premise an initial solution has to be available. Parameters of this initial to be varied can be working principles, working volume as well as working movement.

\section{Spur gear pairs}

Applied to our case the subsystem to be investigated will be pairs of spur gear with different number of teeth combined. 
Starting with the sun and the planet of the initial design draft as the first gear pair the number of teeth of gear 1 and gear 2 was alternately enlarged by one. Holding the pressure angle and the addendum modification coefficients accept for a few boundary values unchanged the module was varied within distinct steps. The smallest value of 39 microns equals half the shank diameter of the end mill being shrank by $22 \%$.

Figure 2 shows the gradients of the transverse contact ratio with respect to increasing module for the different gear pairs. Of central interest is the module value by which each gear pair just reaches the minimum contact ratio of 1 .

To get an idea how this performs geometrically the tendencies of the pitch diameters and the operating pitch diameters were calculated for the gear pairs just fulfilling the minimum demand as expressed above.

The pitch diameter is the product out of the number of teeth and the module and the operating pitch diameter appears additionally considering the addendum modification.

Following Fig. 3 it is obvious that the graphs approach one another as with higher number of teeth the risk for undercut declines and therefore the addendum modification can turn to zero.

It can be recognized that each time the number of teeth is fixed the diameters only vary marginally due to the adjusted addendum modification coefficient. Increasing the number of teeth respectively by one a significant

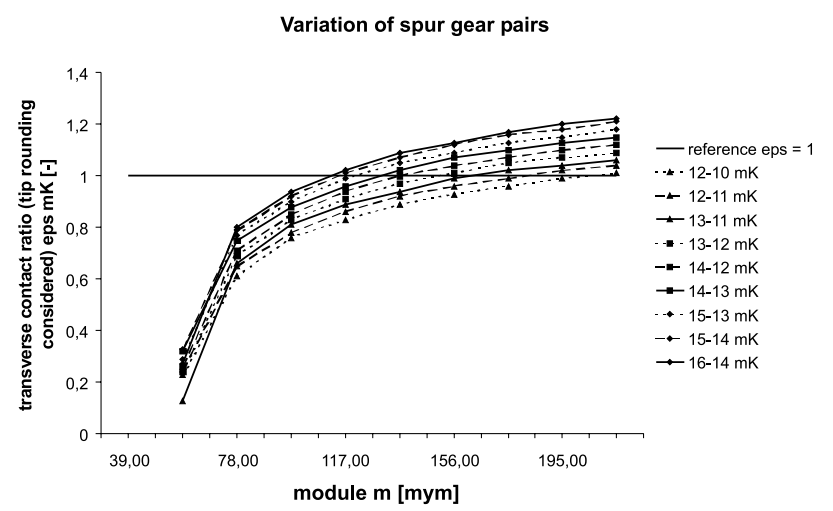

Fig. 2. Variation of spur gear pairs to locate the module reaching the reference of $\varepsilon=1$
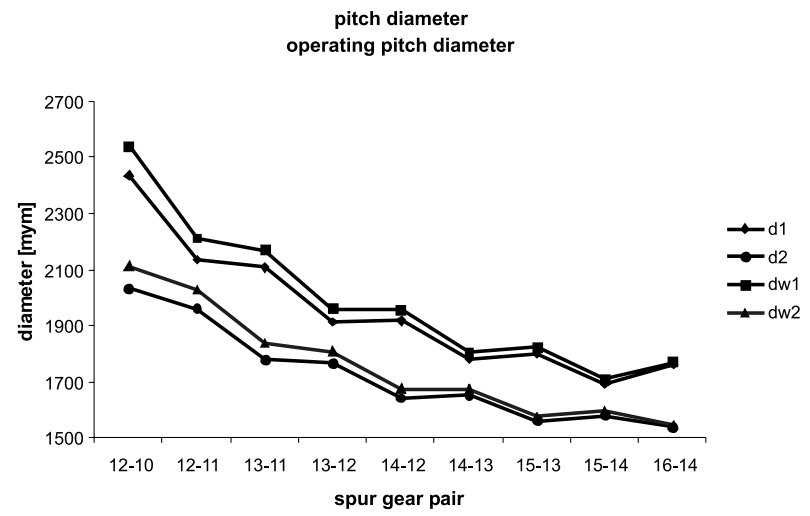

Fig. 3. Diameters on the gear for different spur gear pairs decline occurs which gets less steep with higher number of teeth. This leads towards a countered stage-function.

A first assumption could be drawn: along the objective to find the minimum sized gear drive it seems quite promising to pick a basic spur gear pair where the diameter of one gear decreases with a large delta while the other keeps the same or even declines also. Looking at the over all boundary dimension of the gear pairs (tip side-end of gear 1 to tip side-end of gear 2) the combination 16-14 already increases again in dimension.

But it would be too premature to consider combination 15-14 as the most minimal solution for our demands. So we have to skip to the next higher system level of concretisation.

\section{Planetary gear stages}

To obtain a complete planetary gear stage the ring gear has to be added. Depending on the number of teeth of the sun and the planet the number of teeth will be determined by a condition for the number of teeth [5]. This enables the planet wheels being accurately placed with an angle increment of $120^{\circ}$ around the sun wheel.

Since the calculation of the ring gear diameters also depend on the number of teeth and the module the boundary dimension can change quite a lot.

Moreover, the addendum modification for the ring gears also corresponds with the addendum modifications on the sun and planet. Last but not least, the so far constant pressure angle of commonly used $20^{\circ}$ could also be alternated on behalf of getting a smaller complete system.

The on-going investigation will concentrated on the knowledge found for the combinations 14-12 to 15-14. The combinations below this range have too large addendum modifications on both gears so the centre distances are too far away from the reference centre distances. On the upper end of the scale the combination 16-14 shows the tendency of again increasing dimensions.

\section{3}

\section{Optimised planetary gear layout}

For each of the selected spur gear pairs the lowest number of teeth possible has been calculated for the ring gear. In a start-up configuration the addendum modification coefficients of the sun and the planet were combined with an at first assumed coefficient for the ring gear. Subsequently, the addendum modification coefficients were minimized until undercut occurred or the transverse contact ratio between sun gear and planet gear respectively planet gear and ring gear fell below $\varepsilon=1$.

Having reached the limit of undercut the pressure angle was changed accompanied by repeated variation of the addendum modification coefficients just matching $\varepsilon=1$.

To characterize the later outer measure of the planetary gear stage the parameter 'total boundary dimension' was defined. It consists of the absolute value of the ring root diameter with two times added the height of the chaplet tooth thickness of the ring gear. The graphs of the total boundary dimension for the considered gear stages is presented against the pressure angle in Fig. 4.

Generally speaking, increased pressure angles help in all cases to provide a optimised solution with respect to size. 
Optimized planetary gear drives for miniaturization

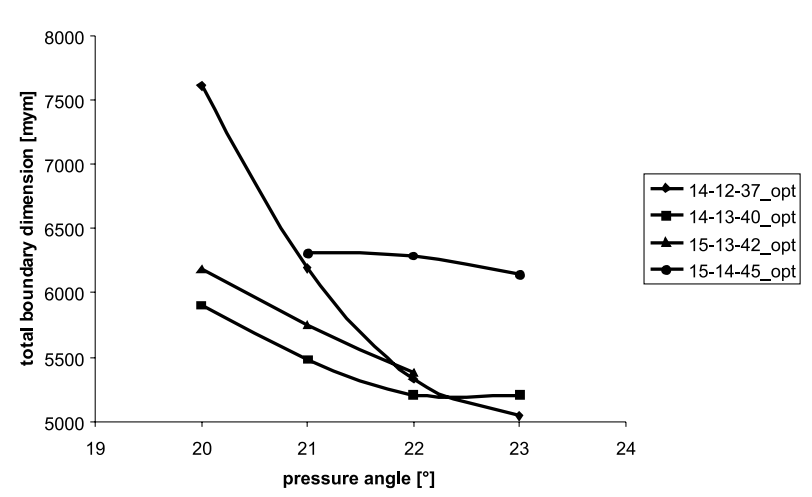

Fig. 4. Total boundary dimension of the planetary gear stages against the pressure angle

Looking solely at gear configurations with standard pressure angle of $20^{\circ}$ the combination 14-13-40 marks the highest miniaturized planetary gear stage with a total boundary dimension marginally below the limit of 6000 microns.

The combination 15-13-42 and the subsequent combination 15-14-45 already cause increasing dimensions. Being primary the worst choice for miniaturization the combination 14-12-37 tremendously declines in its total boundary dimension with increased pressure angle and adapted module. Also the result is decided by a marginal difference the combination 14-12-37 beats the leading combination 14-13-40 right at the end of the examined pressure angle scale.

The ultimate miniaturized and therefore the optimal solution according to the existing restrictions and demands is a planetary gear layout with 14 teeth for the sun, 12 teeth for the planet and -37 teeth for the ring gear in combination with optimised values for the addendum modification coefficients and the pressure angle with at the same minimized module.

\section{4}

\section{Methodology in micro technology}

Looking at the beginning of each product development process the designer first of all has to specify his design task. Subsequently, he collects all demands and restrictions which influence the product. In a lot of cases the designer then ends up with a product design being the initial idea coming to his mind whereas he does not know if it is the most optimal and efficient one. As proven in the conventional mechanical engineering a methodological proceeding helps to find alternative and optimised solutions. The basics for the known methods were constituted when dealing with concrete development problems and verified by many different other applications. Bringing them in a universally valid formulation they are in common use today.

Turning to the micro scale there exists a lack of systematically collected and edited know-how acquired during the concept and draft development of micro mechanical parts and systems that could lead to later on methods. So one important aim of the works carried out by the development of demonstrators is to analyse the methodological proceeding. Formulated as a design manual, effect or principle for this special case it should be examined in forthcoming applications to become finally part of a micro technical method toolbox.

\section{1}

\section{Design manual}

In the case of the systematic variation of different planetary gear drives an important parallel can be realized. Each of the four configurations though having different number of teeth turns to the same spectrum of module after the optimisation of the pressure angle and the addendum modification coefficients. The best solutions 14-12-37 and 14-13-40 both have a module of more or less 116 microns. If we now look at the form tool which implies a measurement makeup for the shrinkage the module of the non-shrank part would be about 150 microns. This equals nearly 1.5 times the shank diameter of the cavity producing micro end mill.

Following this a first design manual can be formulated:

$m(\varepsilon \geq 1)=1.5 * d_{\text {end.mill }}$

This design manual says that in the existing circumstances the module of a miniaturized planetary gear drive just matching a transverse contact ratio of 1 should be about 1.5 times the shank diameter of the end mill used.

\section{5}

According to increased demands for highly chargeable micro mechanical parts and components a micro planetary gear drive has been developed for manufacturing from ceramic materials.

Recruiting the dominant restrictions within the feasible technological parameters for the production of the form tool and the process of ceramic injection moulding (CIM) it was searched for the most miniaturized gear layout.

Since the conception of a planetary gear implies the specification of several different interconnected parameters a systematic way of investigation was recommended. Starting with a simplified focus the optimum of the relevant parameters was found and led step by step to the optimal planetary gear drive in the sense of highest degree of miniaturization.

Looking at the results a design manual could be constituted. This and the fact that the investigation was successfully executed by a systematic variation of existing parameters a methodologically driven product development shows a high potential also in the micro scale.

As the final solution is concerned some conclusions can be drawn:

The decision for the most miniaturized planetary gear stage was finally dependent on the increased pressure angle. This shows how important it is to put all parameters in account. At the same time it reveals that the degree of simplification cannot be driven too far and that intermediate results have to be handled and interpreted carefully with respect to potential influences on the next more concrete step of the total system. 


\section{References}

1. DIN 867 (1986) Basic rack tooth profiles for involute teeth of cylindrical gears for general engineering and heavy engineering

2. Krause W (1993) Konstruktionselemente der Feinmechanik. München, Wien: Carl Hanser

3. Krause W (1996) Zahnradgetriebe für Kleinst- und Mikromotoren. conference Mainz 09./10. may 1996, VDE-VDI-

Gesellschaft Mikroelektronik, Mikro- und Feinwerktechnik, GMM. Düsseldorf: VDI

4. Marz J (2001) Beschränkte Mikrowelt - Restriktionen bei der Konstruktion von Zahnradgetrieben für die Mikrotechnik.
5. Niemann G; Winter M (1983) Maschinenelemente. Volume II, 2nd edition. Springer: Berlin Heidelberg New York Tokyo

6. Roth K (1989) Zahnradtechnik. Volume I, II. Springer: Berlin Heidelberg

7. Thürigen Ch (2000) Zahnradgetriebe für Mikromotoren. Fortschr.-Ber. VDI, Reihe 1, Nr. 326. Düsseldorf: VDI

8. Zimmermann D (2001) Keramische Hochleistungsbauteile in Präzisionsantrieben. Düsseldorf: Springer VDI, Konstruktion 4: $49-50$ 